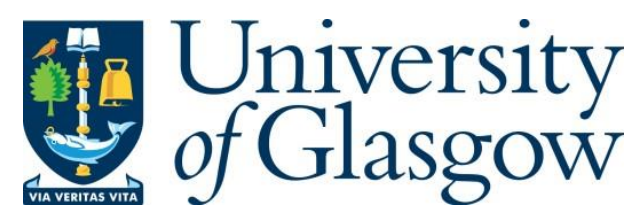

Tong, X., Chang, B., Meng, Z., Zhao, G. and Chen, Z. (2021) Calculating terahertz channel capacity under beam misalignment and user mobility. IEEE Wireless Communications Letters, (doi: 10.1109/LWC.2021.3127884).

There may be differences between this version and the published version. You are advised to consult the publisher's version if you wish to cite from it.

$\underline{\text { http://eprints.gla.ac.uk/258627/ }}$

Deposited on: 8 November 2021

Enlighten - Research publications by members of the University of Glasgow http://eprints.gla.ac.uk 


\title{
Calculating Terahertz Channel Capacity under Beam Misalignment and User Mobility
}

\author{
Xin Tong, Bo Chang, Zhen Meng, Guodong Zhao, and Zhi Chen
}

\begin{abstract}
In this letter, we consider the terahertz (THz) wireless communication between an unmanned aerial vehicle (UAV) and a base station. The beam alignment is interrupted by some unpredictable factors, such as air turbulence, and the UAV recovers the beam alignment via flying maneuver. Here, we propose a new method to obtain the closed-form channel capacity under the beam misalignment, where a classical control model is used to capture the trajectory of the UAV maneuver. This allows us to obtain a much accurate channel capacity compared with the conventional methods that treat the misalignment as a random variable. The numerical results show that the proposed method reduces almost $80 \%$ calculation error compared to the conventional method.
\end{abstract}

Index Terms-Channel capacity, beam misalignment, THz communication.

\section{INTRODUCTION}

Beam misalignment is critical for wireless communication systems that operate on high frequency bands, e.g., terahertz (THz) communication systems [1]. For mobile platforms, e.g., an unmanned aerial vehicle (UAV), it is a natural choice to use their mobility to have a good alignment between the transmitter and receiver. For example, as shown in Fig. 1, where a UAV is communicating with a base station, the basic idea is to control the UAV to fly to a desired position. In this way, the UAV can enjoy an excellent channel capacity. In the following of this paper, the mobile UAV scenario is adopted as a typical example to demonstrate our method, which can be extended into other mobile scenarios straightforwardly, e.g., autonomous vehicles in V2X networks and automatic guided vehicles in smart factories.

Beam misalignment can significantly reduce data rate in $\mathrm{THz}$ communications, especially considering mobility scenario. For example, for a Cassegrain antenna working at 220 $\mathrm{GHz}$, the effective beamwidth is about $0.3^{\circ}$ [2]. If an UAV is moving with velocity about $60 \mathrm{~km} / \mathrm{h}$ and about $50 \mathrm{~m}$ distance from the transmission antenna, the effective beamwidth is about $2 \mathrm{~m}$, which can be only covered in about one hundred millisecond (ms) with such high velocity of UAV. Then, the

Manuscript received May 24, 2021; revised October 13, 2021; accepted November 5 2021. This work was supported by the National Natural Science Foundation of China under Grant 61631004. The associate editor coordinating the review of this article and approving it for publication was Prof. Jinho Choi. (Corresponding author: Bo Chang.)

X. Tong, B. Chang, and Z. Chen are with the National Key Lab. on Communications, University of Electronic Science and Technology of China (UESTC), Chengdu, 611731, China (e-mail: tongx1990@163.com; changb3212@163.com; chenzhi@uestc.edu.cn).

Z. Meng and G. Zhao are with the School of Engineering, University of Glasgow, Glasgow, G12 8QQ, UK (e-mail: mengzhen@@student.gla.ac.uk Guodong.Zhao@glasgow.ac.uk).

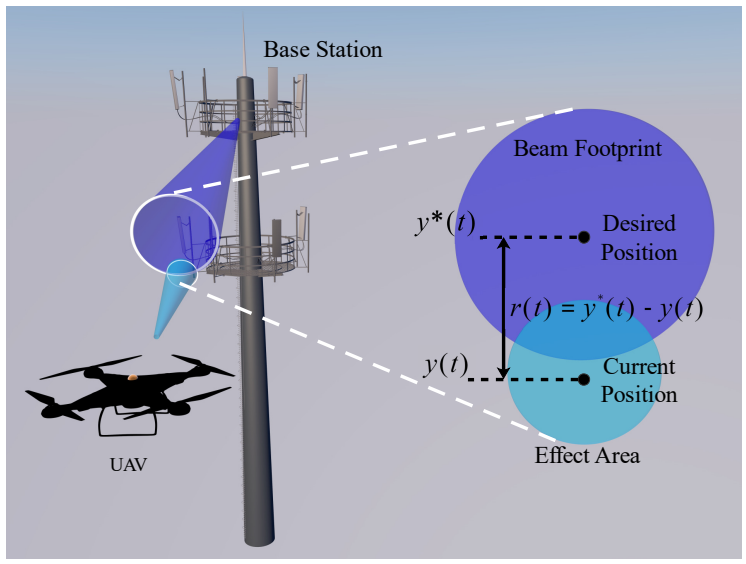

Fig. 1. A typical THz communication scenario where a UAV flies from $y(t)$ to $y^{*}(t)$ to recover the beam alignment.

beam alignment and data transmission should be finished in the level of one hundred ms. Otherwise, the UAV would be out of the effective beamwidth. Then, it is very difficult to align beam and transmit data in such limited time.

Currently, there are many research papers [3] [4] working on UAV path planning for beam alignment. However, the beam misalignment would still happen in practice due to many imperfections, for example, the dynamics of the environment and mobile platform. In order to take the beam misalignment into account when calculating the channel capacity, most existing works [5] [6] treat the misalignment as a random variable, like Rayleigh fading, and choose different values of the variance to capture the beam misalignment. For example, if the weather condition is poor or the UAV has poor flying performance, a large variance should be used. In contrast, if the weather condition is good and the UAV has excellent flying performance, a small variance should be selected. However, this kind of methods usually suffers from either overestimating or underestimating the channel capacity due to the large dynamics of the environment and mobile platform [7].

In this letter, we propose a new method to calculate the channel capacity under beam misalignment, but from another perspective where the mobility of the mobile platform is modeled as a classical control process. Specifically, as shown in Fig. 1 again, we consider a typical case where a UAV hovers at a desired position to maintain the beam alignment between the antenna mounted on the UAV and the antenna mounted at the base station tower. The unpredictable air turbulence blows the UAV away from the desired position and causes the beam misalignment. Then, the UAV recovers the beam 


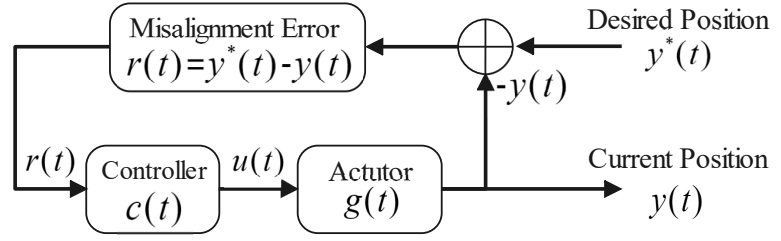

Fig. 2. The classical control model in UAV operation.

alignment via its mobility, i.e., flying back to the desired position. Here, we propose to use the classical control model to capture the detailed flight trajectory. As a result, a much accurate channel capacity can be obtained compared with the conventional method that treats misalignment as a random variable.

\section{Wireless Channel Model and Classical CONTROL MODEL}

\section{A. Wireless Channel Model in THz Frequency Band}

According to [5], the $\mathrm{THz}$ wireless channel at the time $t$ can be modeled as

$$
h(t)=h_{l} h_{f} h_{p}(t),
$$

where $h_{l}$ and $h_{f}$ represent path loss and multi-path fading, respectively. The term $h_{p}(t)$ is the misalignment fading at the time $t$ and can be expressed by

$$
h_{p}(t)=A_{0} \exp \left(-\frac{2 r(t)^{2}}{w_{e q}^{2}}\right)
$$

where $A_{0}$ and $w_{e q}^{2}$ are constant parameters ${ }^{1}$, and $r(t)$ is the misalignment error, defined as the radial distance between the two beams' centers shown in Fig. 1 [5]. $y(t)$ and $y^{*}(t)$ denote the desired and current positions of the UAV, respectively, i.e.,

$$
r(t)=y^{*}(t)-y(t) .
$$

The channel capacity with unit bandwidth is expressed by

$$
C_{\text {ins }}(t)=\log _{2}\left(1+\frac{p|h(t)|^{2}}{N_{0}}\right),
$$

where $p$ is the transmitter power, and $N_{0}$ is the noise power. According to Jensen's inequality in [8], the ideal average channel capacity $C_{i d}$ is upper bounded by

$$
C_{i d} \leq \log _{2}\left(1+\frac{p_{0}}{N_{0}} \mathbb{E}\left\{\left|h_{l}\right|^{2}\left|h_{f}\right|^{2}\right\} \mathbb{E}\left\{\left|h_{p}(t)\right|^{2}\right\}\right) .
$$

\section{B. Classical Control Model in UAV Operation}

When the UAV flies from its current position to the desired position to re-align the beams, we use the classical control model to capture the recovery trajectory. This is because the UAV is actually a control system and follows the basic control laws.

\footnotetext{
${ }^{1} A_{0}$ is the fraction of the collected power at $r(t)=0$, and $w_{e q}^{2}$ indicates the equivalent beam-width, which is only related to the distance between the transmitter and the receiver [5].
}

As shown in Fig. 2, the misalignment error $r(t)$ is first calculated by the difference between the UAV's current position and the desired position. Then, the control command is generated via a control algorithm $c(t)$, i.e.,

$$
u(t)=c(t) \otimes r(t)
$$

where $\otimes$ is the convolution operation. Once the control command $u(t)$ is executed via a motor $g(t)$ (also called actuators in control systems), the position of the UAV $y(t)$ is changed accordingly, i.e.,

$$
y(t)=u(t) \otimes g(t) .
$$

As the above control process continues, the misalignment error $r(t)$ reduces, which makes the UAV fly towards the desired position $y^{*}(t)$.

\section{Channel Capacity in Beam Alignment RECOVERY}

In this section, we calculate the channel capacity when the UAV is conducting beam alignment recovery by flying from its current position to the desired one. Specifically, we first obtain the expression of the misalignment fading, which allows us to have the instant channel capacity. Then, we take the integral operation over the misalignment fading and obtain the average capacity during the beam alignment recovery.

\section{A. Instant Capacity}

We use the classical control theory to obtain the approximation expression of the alignment error $r(t)$. Specifically, we denote $G(s), C(s), Y(s), Y^{*}(s)$, and $R(s)$ as the Laplace transforms of $g(t), c(t), y(t), y^{*}(t)$, and $r(t)$, then we have

$$
R(s)=\frac{1}{1+C(s) G(s)} Y^{*}(s)=\frac{1}{a_{n}\left(s-x_{1}\right)\left(s-x_{2}\right) \ldots\left(s-x_{r}\right)},
$$

where $X=\left\{x_{1}, x_{2}, \ldots, x_{r}\right\}$ is known as the poles of the control system. Using $\mathbb{L}^{-1}[\cdot]$ to denote the inverse Laplace operator, we have

$$
r(t)=\mathbb{L}^{-1}[R(s)]=\sum_{i=1}^{r} C_{i} e^{\mathfrak{R}\left\{x_{i}\right\} t} \cos \left(\mathfrak{J}\left\{x_{i}\right\} t\right),
$$

where the parameters $C_{i}$ are obtained from Example 2.2 in [9], and $\mathfrak{R}\left\{x_{i}\right\}$ and $\mathfrak{I}\left\{x_{i}\right\}$ represent the real and imaginary part of $x_{i}$, respectively. If we rewrite the roots in the following order $\mathfrak{R}\left\{x_{1}\right\} \leq \mathfrak{R}\left\{x_{2}\right\} \leq \ldots \leq \mathfrak{R}\left\{x_{i}\right\} \leq \ldots \leq \mathfrak{R}\left\{x_{r}\right\}$, the root $x_{r}$ is the rightmost pole, also called the dominant pole. It means that the term with $x_{r}$ in (8) dominates the value of $r(t)$. As a result, we can use the dominant term to represent $r(t)$, i.e.,

$$
r(t) \approx C_{r} e^{\mathfrak{R}\left\{x_{r}\right\} t} \cos \left(\mathfrak{J}\left\{x_{r}\right\} t\right) .
$$

This is also called the dominant pole approximation in [10]. According to the classical control theory in [9], when $x_{r}$ is sufficiently closer to the Y-axis (Laplace domain) than the other poles, this approximation error is very low. Thus, it is popular to adopted the approximation to simplify system 


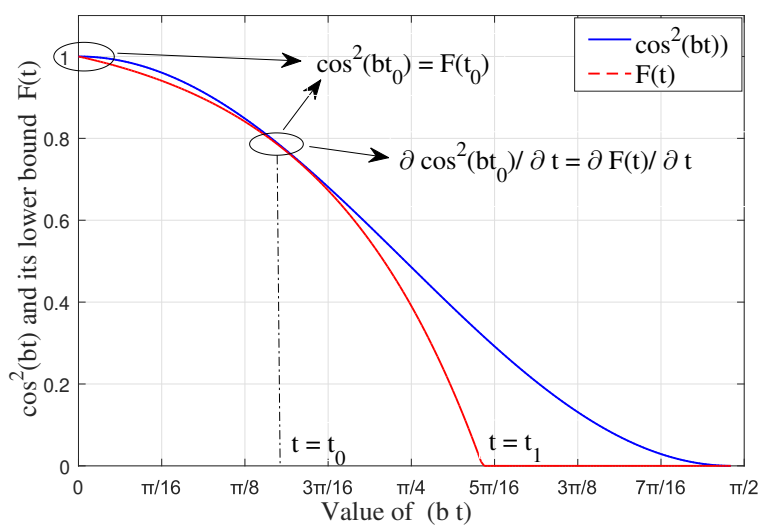

Fig. 3. A case that $\cos ^{2}(b t)$ is lower bounded by $F(t)$, where $G(s)=$ $1 /(s+1)$, and $C(s)=2+100 / s$. The dominant pole of such a control system can be obtained as $x_{r}=-1.3+6.98$, i.e., $a=-1.3$ and $b=6.98$.

analysis from motion control perspective [10]. Substituting (9) into (2), we obtain the instant misalignment fading as follows,

$$
h_{p}(t)=A_{0} \exp \left(-\frac{2 C_{r}^{2} e^{2 \mathfrak{R}\left\{x_{r}\right\} t} \cos ^{2}\left(\mathfrak{J}\left\{x_{r}\right\} t\right)}{w_{e q}^{2}}\right) .
$$

By substituting (10) into (4), we obtain the instant channel capacity

$$
C_{\text {ins }}(t)=\log _{2}\left(1+\frac{p_{0}}{N_{0}}\left|h_{l}\right|^{2}\left|h_{f}\right|^{2}\left|h_{p}(t)\right|^{2}\right) .
$$

\section{B. Average Capacity}

Next, we calculate the upper bound $\bar{C}(T)$ of the average capacity in (5) for the period of 0 to $T$ seconds. Specifically, from (10), we have

$$
\mathbb{E}\left[\left|h_{p}(t)\right|^{2}\right]=\frac{A_{0}^{2}}{T} \int_{0}^{T} \exp \left(\frac{-4 C_{r}^{2}}{w_{e q}^{2}} e^{2 \mathfrak{R}\left\{x_{r}\right\} t} \cos ^{2}\left(\mathfrak{J}\left\{x_{r}\right\} t\right)\right) d t .
$$

To calculate the above integration, we simplify (12) by using a piece-wise function to construct the lower bound of the term $\cos ^{2}\left(\mathfrak{J}\left\{x_{r} t\right\}\right)$. Then, we take the lower bound back into (12) and obtain an upper bound capacity.

1) Simplifying (12): We use $a$ to denote $\mathfrak{R}\left\{x_{r}\right\}$ and consider the case $a<0$, which is corresponding to a stable control system [9]. We use $b$ to denote $\mathfrak{I}\left\{x_{r}\right\}$ and consider the case $b \geq 0$ since $b$ only appears in cos function and negative $b$ has the same result as positive $b$. We replace the term $\cos ^{2}(b t)$ by using the following inequality,

$$
\cos ^{2}(b t) \geq F(t) \stackrel{\text { Def }}{=} \begin{cases}A-B e^{-2 a t}, & \text { for } t \leq t_{1}, \\ 0, & \text { for } t>t_{1} .\end{cases}
$$

In the following, we find the values of $A, B, t_{1}$ in (13) to let $\cos ^{2}(b t)$ and $F(t)$ as close as possible.

As shown in Fig. 3, due to the fact that a) $F(t)=\cos ^{2}(b t)$ holds when $t=0$ and $t=t_{0}$, b) $\partial F(t) / \partial t=\partial \cos ^{2}(b t) / \partial t$ holds when $t=t_{0}$, and c) $F(t)=0$ holds when $t=t_{1}$, we obtain the following expressions:

$$
\begin{aligned}
& A=B+1, \\
& \cos ^{2}\left(b t_{0}\right)=A-B e^{-2 a t_{0}}, \\
& -2 b \sin \left(b t_{0}\right) \cos \left(b t_{0}\right)=2 a B e^{-2 a t_{0}}, \\
& A-B e^{-2 a t_{1}}=0
\end{aligned}
$$

where $t_{0} \in[0, \pi /(2 b)]$. Combining the above four expressions, we obtain the following two expressions

$$
\begin{aligned}
& B=\frac{\cos ^{2}\left(b t_{0}\right)-1}{1-e^{-2 a t_{0}},} \\
& t_{1}=-\frac{\log \left(\frac{A}{B}\right)}{2 a},
\end{aligned}
$$

where $t_{0}$ is the solution of the expression

$$
\frac{a}{b} \tan \left(b t_{0}\right)+1-e^{2 a t_{0}}=0 .
$$

Here, $t_{0}$ can be obtained by solving (20) since it is a uni-variate function. Substituting $t_{0}$ into (14), (18), and (19), the values of $A, B, t_{1}$ can be obtained, i.e., we obtain the simplified upper bound expression of $\mathbb{E}\left[\left|h_{p}\right|^{2}\right]$ as follows,

$$
\mathbb{E}\left[\left|h_{p}\right|^{2}\right] \leq \frac{A_{0}^{2}}{T} \int_{0}^{T} \exp \left(\frac{-4 C_{r}^{2}}{w_{e q}^{2}} e^{2 a t} F(t)\right) d t .
$$

2) Calculating Capacity Upper Bound: According to (13), the expression of the misalignment fading (21) includes only one segment if $T \leq t_{1}$. Otherwise, it includes two segments if $T>t_{1}$. Specifically, when $T \leq t_{1}$, we have

$$
\begin{aligned}
\mathbb{E}\left[\left|h_{p}\right|^{2}\right] \leq & \frac{A_{0}^{2}}{T} \int_{0}^{T} e^{-4 A C_{r}^{2} e^{2 a t} / w_{e q}^{2}} \times e^{4 B C_{r}^{2} / w_{e q}^{2}} d t \\
& \stackrel{z=e^{2 a t}}{\underline{A_{0}^{2} \exp \left(4 B C_{r}^{2} / w_{e q}^{2}\right)}} \frac{e_{1}^{2 a T} \frac{e^{-4 A C_{r}^{2} z / w_{e q}^{2}}}{z} d z}{2 a T} d-\operatorname{Ei}\left[-4 A C_{r}^{2} / w_{e q}^{2}\right] \\
= & \left.\quad+\operatorname{Ei}\left[-4 A C_{r}^{2} / w_{e q}^{2} \times e^{2 a T}\right]\right) \\
& \stackrel{A_{0}^{2} \exp \left(4 B C_{r}^{2} / w_{e q}^{2}\right)}{2 a T}\left(H_{1}(T),\right.
\end{aligned}
$$

where $\operatorname{Ei}[\cdot]$, known as the exponential integral, is defined by

$$
\operatorname{Ei}[x]=-\int_{t=-x}^{\infty} \frac{e^{-t}}{t} d t
$$


When $T>t_{1}$, we have

$$
\begin{aligned}
\mathbb{E}\left[\left|h_{p}\right|^{2}\right] & =\frac{1}{T}\left\{H_{1}\left(t_{1}\right) t_{1}+\frac{A_{0}^{2}}{T} \int_{t_{1}}^{T} \exp \left(\frac{-4 C_{r}^{2}}{w_{e q}^{2}}\right) d t\right\} \\
& =\frac{t_{1}}{T} H_{1}\left(t_{1}\right)+\frac{A_{0}^{2}\left(T-t_{1}\right)}{T} \exp \left(\frac{-4 C_{r}^{2}}{w_{e q}^{2}}\right) \\
& \stackrel{\text { Def }}{=} H_{2}(T) .
\end{aligned}
$$

Combining (5), (22), and (24), we obtain the upper bound of the average channel capacity as follows,

$$
\bar{C}(T)= \begin{cases}\log _{2}\left(1+\frac{p_{0}}{N_{0}} \mathbb{E}\left\{\left|h_{l}\right|^{2}\left|h_{f}\right|^{2}\right\} H_{1}(T)\right), \text { for } & T \leq t_{1}, \\ \log _{2}\left(1+\frac{p_{0}}{N_{0}} \mathbb{E}\left\{\left|h_{l}\right|^{2}\left|h_{f}\right|^{2}\right\} H_{2}(T)\right), \text { for } \quad T>t_{1} .\end{cases}
$$

\section{NuMERICAL RESULTS}

Since we focus on the impact of the misalignment fading $h_{f}$ on channel capacity, we assume $\mathbb{E}\left\{\left|h_{l}\right|^{2}\right\}=1$ and $\mathbb{E}\left\{\left|h_{f}\right|^{2}\right\}=1$ for simplicity in this section. According to [5], we adopt the parameters of misalignment fading as $A_{0}=1.1$ and $W_{e q}=1.3$. We further consider the typical proportional-integral controller with a simple first-order actuator [9], where

$$
C(s)=P+\frac{I}{s}, \quad G(s)=\frac{1}{s+1}
$$

with $P=2$, and $I=2,20$.

Fig. 4 illustrates the instant capacity performance during the beam recovery, where the initial misalignment error is $r(0)=1$ $\mathrm{m}$ in this example. Here, we consider two typical control processes. The first one with the control parameter $I=2$ makes the UAV monotonically fly back to the desired position. The second one with the control parameter $I=20$ makes the UAV fly back to the desired position with oscillation. From the results, the theoretical curves match the simulation ones very well, verifying the approximations in (9).

Fig. 5 demonstrates the performance of the average capacity error $C_{e}$ versus the standard deviation used in the conventional method. For the average capacity over the first 2000 milliseconds, the conventional method has the lowest capacity error with $\delta=0.2$. When we look at the first 5000 milliseconds case, the optimal $\delta=0.15$. In particular, there is a cross-over point between the curves with conventional methods. The is because when $\delta=0$, the traditional method has an overestimation on the capacity. While it has an underestimation when $\delta$ is sufficiently large. Thus, it is hard for the conventional method to have good performance over different periods of time. This is reasonable since the conventional method fails to capture the trajectory details.

Again in Fig. 5, since the proposed method is developed from a motion control perspective, instead of choosing the value of $\delta$, the red curves are constant. Additionally, it also enjoys much better performance in all cases, since the control model captures the detailed trajectory and precisely calculates the capacity at each point. For example, the proposed method significantly reduces the estimation error by $80 \%$ (i.e., from $0.03 \mathrm{Bit} / \mathrm{s} / \mathrm{Hz}$ to $0.006 \mathrm{Bit} / \mathrm{s} / \mathrm{Hz}$ ) in $T=2000 \mathrm{~ms}$ case, and

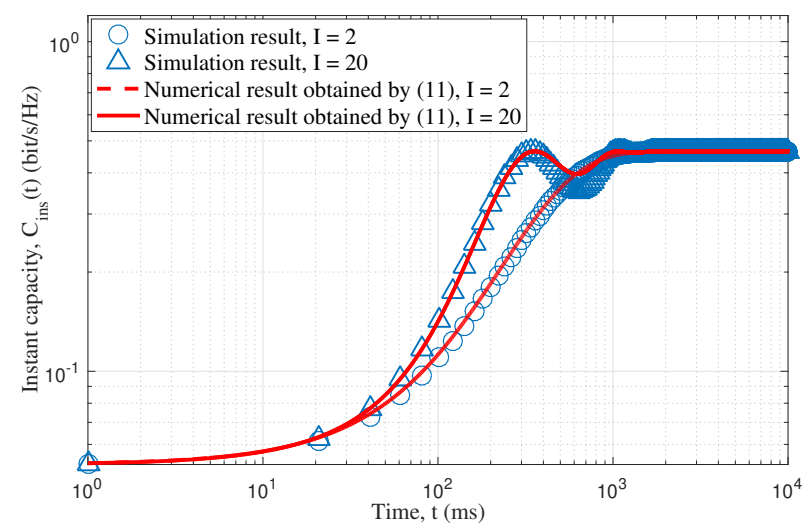

Fig. 4. Instant capacity under different control parameters. The simulation results are obtained based on (4), (6), (7), and (26). The numerical results are obtained by the closed-form expressions (11).

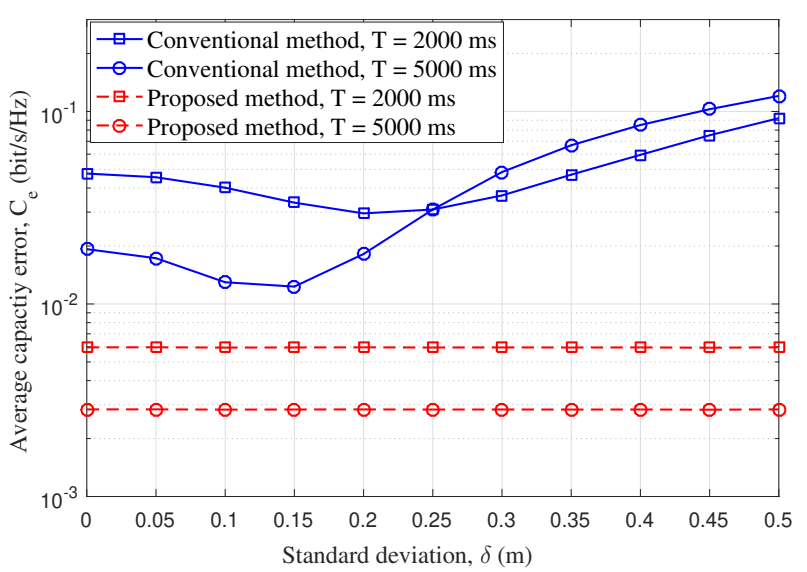

Fig. 5. Average capacity error $C_{e}$ over different time periods $T$. Here, we define $C_{e}=\left(C_{1}+C_{2}+\ldots+C_{M}\right) / M$, where $\left\{C_{1}, C_{2}, \ldots, C_{m}, \ldots, C_{M}\right\}$ are the capacity error, obtained by the Monte Carlo method with $M=1000$. The capacity error $C_{m}$ is obtained by $C_{m}=\left|C_{m}^{\#}-C_{m}^{*}\right|$, where $C_{m}^{*}$ is the simulation capacity, and $C_{m}^{\#}$ is the calculation capacity. $C_{m}^{\#}$ is obtained by the conventional method [5] and the proposed expression (25), respectively.

$75 \%$ (from $0.012 \mathrm{Bit} / \mathrm{s} / \mathrm{Hz}$ to $0.003 \mathrm{Bit} / \mathrm{s} / \mathrm{Hz}$ ) in $T=5000 \mathrm{~ms}$ case, respectively.

\section{Conclusions}

This letter considered the terahertz UAV communications and proposed a method to calculate the average capacity under the misalignment fading during the beam alignment recovery. Compared with the conventional methods, the proposed method captured the misalignment error from a classical control perspective, which obtained a more accurate capacity. The numerical results showed that the proposed method the proposed method reduces almost $80 \%$ calculation error compared to the conventional method. In the future, we will extend this work to more complicated scenarios, such as mobile to mobile cases. 


\section{REFERENCES}

[1] K. J. Kaltenecker, E. Kelleher, B. Zhou, et al., "Attenuation of THz beams: A 'how to' tutorial," Journal of Infrared, Millimeter, and Terahertz Waves, vol. 40, no. 8, pp. 878-904, Jul. 2019.

[2] Z. Chen, et al., "A survey on terahertz communications," China Сотmunications, vol. 16, no. 2, pp. 1-35, Feb. 2019.

[3] S. Li, B. Duo, X. Yuan, Y. Liang, and M. D. Renzo, "Reconfigurable intelligent surface assisted UAV communication: Joint trajectory design and passive beamforming," IEEE Wireless Communications Letters, vol. 9, no. 5, pp. 716-720, May 2020.

[4] Y. Pan, K. Wang, C. Pan, H. Zhu, and J. Wang, "UAV-assisted and intelligent reflecting surfaces-supported terahertz communications," IEEE Wireless Communications Letters, vol. 10, no. 6, pp. 1256-1260, Jun. 2021.

[5] A. A. Boulogeorgos, E. N. Papasotiriou, and A. Alexiou, "Analytical performance assessment of THz wireless systems," IEEE Access, vol. 7, pp. 11436-11453, Jan. 2019.

[6] J. Kokkoniemi, A. A. Boulogeorgos, M. Aminu, et al., "Impact of beam misalignment on THz wireless systems," Nano Communication Networks, vol. 24, p. 100302, May 2020.

[7] S. K. Moorthy and Z. Guan, "LeTera: Stochastic beam control through ESN learning in terahertz-band wireless UAV networks," in Proc. IEEE INFOCOM 2020, Toronto, ON, Canada, Jul. 2020, pp. 1039-1044.

[8] S. G. Krantz, Handbook of Complex Variables, Springer, 1999.

[9] R. C. Dorf and R. H. Bishop, Modern Control Systems, 13th ed, Boston, 2016.

[10] P. V. Mieghem, "The asymptotic behavior of queueing systems: Large deviations theory and dominant pole approximation," Queueing Systems, vol. 23, no. 1, pp: 27-55, Mar. 1996. 